\title{
Intraluminal Pressure Difference of Bakri Balloon in the positive Tamponade test During the Management of severe Post-Partum Hemorrhage
}

\author{
Hong $\mathrm{Li}^{1}$, Tong Yu Bai ${ }^{2}$, Liang Bai*3, Rong Li Yang ${ }^{4}$, Xiao Guang Shao ${ }^{1}$ and Xiuhua Xu ${ }^{1}$ \\ ${ }^{1}$ Department of Obstetric, The Women and Children Center of Da Lian, China \\ ${ }^{2}$ The Thirteenth High Middle School of Da Lian, China \\ ${ }^{3}$ Department of Oral Maxillofacial Surgery, China \\ ${ }^{4}$ ICU Department, China
}

*Corresponding author: Liang Bai, Department of Oral Maxillofacial Surgery, China

\begin{abstract}
ARTICLE INFO
Received: 蔧 February 02, 2019

Published: 幽 March 08, 2019

Citation: Hong Li, Tong Yu Bai, Liang Bai, Rong Li Yang, Xiao Guang Shao, et al., Intraluminal Pressure Difference of Bakri Balloon in the positive Tamponade test During the Management of severe Post-Partum Hemorrhage. Biomed J Sci \& Tech Res 15(4)-2019. BJSTR. MS.ID.002735.
\end{abstract}

Keywords: Intraluminal Pressure; Balloon Tamponade; Postpartum Hemorrhage; Tamponade Test

\section{ABSTRACT}

Objective: To determine the difference of the intraluminal pressure of Bakri balloon in vitro and after the establishment of a positive 'tamponade test' in the management of severe postpartum hemorrhage.

Materials and Methods: A DigiMano pressure recorder was attached via a three-way tap to a Bakri balloon. Intraluminal pressure readings (ILPs) were recorded after each $50-\mathrm{ml}$ aliquot of normal saline had been insufflated into the balloon ex vitro. During the treatment of postpartum hemorrhage, the corresponding ILPs and blood pressure were recorded after the positive tamponade test was obtained. The difference value of the ILPs in vivo and in vitro was figured out and compared.

Results: After the positive tamponade test was established during the treatment of postpartum hemorrhage, the ILPs in Bakri balloon increased by the value of $39 \pm 12 \mathrm{mmHg}$.

Conclusion: After a positive tamponade test was established, the intraluminal pressure within the tamponade balloon increased in vivo than in vitro, the difference value was roughly equal to the uterine spiral artery vasculature pressure.

\section{Introduction}

Bakri balloon tamponade has been widely used in the management of postpartum hemorrhage recently, with the efficacy is $70 \%-90 \%$ [1]. The proposed mechanism is by creating an intrauterine pressure, including uterine shape changes, secondary uterine activity, balloon-endometrial interactions and distal effects on the flow within the uterine arteries [2,3]. A 'tamponade test' is used to determine the clinical effectiveness [3]. There are a few papers involved the measurement of luminal pressure of a variety of balloons in vitro or in vivo. C Georgiou ${ }^{[2]}$ detected pressure generated by the Bakri balloons in two postpartum hemorrhage patients, he got the conclusion that intraluminal pressure within tamponade balloon did not exceed the systolic blood pressure of the patient when a positive tamponade test was established. In our article, we detected and monitored the intrauterine intraluminal pressure (ILPs) within a Bakri balloon every two hours after achieving a positive tamponade test in 30 postpartum hemorrhage patients , recorded the patients' blood pressure and the amount of blood loss, measured and compared the difference of the ILPs in vivo and in vitro, the aim was to find the optimal inflation liquid volume of filling the Bakri balloon to establish a positive tamponade test, and to explain the working rationale of Bakri balloon about intraluminal pressure.

\section{Materials and Methods}

Setting: The obstetric department in the Center of Children and Women (7,000 births) in Da Lian city, Liao Ning province, China. Sample: 30 women who required a Bakri balloon and (or) other 
invasive surgical techniques to control postpartum hemorrhage. The ethics committee approval in the hospital was required.

\section{In vitro ILPs in the Bakri Balloon}

The ILPs were recorded for the Bakri balloon in the laboratory setting. These readings were obtained using a pressure a Digi Mano (Netech Corp oration, Farmingdale, NY, USA) recorder, which was used widely in clinic medicine. The ILPs were recorded after 50 $\mathrm{ml}$ aliquots of normal saline had been used to insufflate the Bakri balloon until a final volume of $600 \mathrm{ml}$ was reached. Five separate series (0-600 $\mathrm{ml}$ in 50-ml aliquots) were recorded from five different Bakri balloons.

\section{ILPs in the Bakri Balloon after Positive Tamponade Test Established}

From January 2016 to January 2018, patients with massive PPH (blood loss $\geq 1500 \mathrm{ml}$ ) who needed intrauterine Bakri balloon insertion with (without) other hemostasis techniques were included. After the positive tamponade test established, the ILPs within the Bakri balloon were detected every two hours until the balloon was removed. The cause of the PPH, the sequence of treatments, estimated total blood loss and clinical outcomes were recorded. The procedure for insertion of the Bakri balloon was similar to that described by the inventor [4], vaginal packing was performed when there was evidence of slippage or prolapse of the balloon through the cervix [4]. The distal end of the catheter was tapped to the patient's thigh, but weights were not used. Ultrasound visualization of balloon placement was necessary in our series [5]. When the balloon was inserted during a caesarean section, the distal end of the balloon shaft was passed through the cervical opening with an assistant pulling the end per vagina. If bleeding was arrested after balloon inflation, the balloon tamponade was continued for 24 hours, after which, the deflation was performed. All patients had an indwelling Foley catheter to monitor urine output, received continuous syntocine for 24 hours until the Bakri balloon was deflated. Broad spectrum antibiotics were used for prophylaxis.

\section{Statistical Procedures}

The statistical analysis was performed using SPSS software(version 16; SPSS Inc, Chicago, IL).Continuous data were presented using means and standard deviation (SDs)

\section{Results}

\section{In vitro ILPs in the Bakri balloon}

The readings demonstrated that in absence of any external restrictions, ILPs increased slowly by the increase of the amount of filled saline liquid, reached to the peak of $60-70 \mathrm{mmHg}$ at 600 $\mathrm{ml}$ insufflation. There is no fluctations in ILP when the balloon was allowed to stand for 2 hours. when an external compressive force was applied, such as squeezing the balloon by hand, the ILP increased as expected. The numerical relationship between this volume and the resulting ILP is linear, the linear regression model is $\mathrm{y}=31.033+0.768 \mathrm{x}, \mathrm{y}$ : intraluminal pressure, $\mathrm{x}$ : liquid volume.

\section{Bakri Intrauterine Balloon Tamponade in the Management of Severe Postpartum Hemorrhage Patients}

There were 14,886 deliveries in our department during the period. The frequency of primary PPH with an estimated blood loss exceeding $500 \mathrm{ml}$ was $3.6 \%(\mathrm{n}=535)$. The frequency of massive $\mathrm{PPH}$ with an estimated blood loss exceeding $1.5 \mathrm{~L}$ was $0.55 \%$ $(\mathrm{n}=82)$. Among the 82 patients with massive $\mathrm{PPH}, 30$ cases were used with Bakri balloon catheter. During the same period, there were 5 peripartum hysterectomies, 2 involved attempt ed use of the Bakri balloon catheter and were not included in this case series. All the identified cases of PPH that involved use of the Bakri balloon catheter failed initial medical management with oxytoxics, including bolus syntometrine, syntocinon bolus and infusion, and intra muscular carboprost injections. In 18 cases, the Bakri balloon was the only modality of treatment after medical management; in the remaining 12 patients, it was used combined with uterine compression sutures or pelvic artery ligature or pelvic artery embolization. All patients were transferred to a High Dependency Unit, some required transfusion with blood cells or fresh frozen plasma. There were no major complications arising from the use of the Bakri balloon catheter.

Table 1: The intraluminal and blood pressure readings obtained in 30 cases $(n=30)$.

\begin{tabular}{|c|c|c|c|c|c|c|}
\hline $\begin{array}{c}\text { Estimated } \\
\text { Blood Loss } \\
\text { (ml) }\end{array}$ & $\begin{array}{c}\text { Volume in Balloon } \\
\text { at Positive } \\
\text { Tamponade Test } \\
\text { (ml) }\end{array}$ & $\begin{array}{c}\text { Intraluminal } \\
\text { Pressure (ILP) } \\
\text { (mmH) }\end{array}$ & $\begin{array}{c}\text { Systolic } \\
\text { Pressure } \\
\text { (SP) } \\
\text { (mmHg) }\end{array}$ & $\begin{array}{c}\text { Diastolic Pressure } \\
\text { (DP) } \\
\text { (mmHg) }\end{array}$ & $\begin{array}{c}\text { Difference } \\
\text { between ILP and } \\
\text { DP } \\
\text { (mmHg) }\end{array}$ & $\begin{array}{c}\text { ILP Difference } \\
\text { Between } \text { in } \\
\text { vivo } \text { and } \text { in } \\
\text { vitro(mmHg) }\end{array}$ \\
\hline $2720 \pm 1075$ & $405 \pm 47$ & $95 \pm 13$ & $111 \pm 13$ & $65.4 \pm 9.8$ & $29 \pm 15$ & $39 \pm 12$ \\
\hline
\end{tabular}

\section{ILPs of Bakri Balloon in the PPH Patients}

Following the establishment of a positive tamponade test, the saline liquid volume in balloon was $405 \pm 47 \mathrm{ml}$, Intraluminal pressure (ILP) in Bakri balloon was $95 \pm 13 \mathrm{mmHg}$, which is higher than in vitro, the difference is $39 \pm 12 \mathrm{mmHg}$. ILPs in Bakri balloon did not show obvious fluctuation during the patient bed rest but increased when extra forces applied to the patient's abdomen, such as cough, uterine massage. Patients' average systolic pressure were $111 \pm 13 \mathrm{mmHg}$, diastolic pressure was $65.4 \pm 9.8 \mathrm{mmHg}$, which revealed that the actual ILPs in Bakri did not exceed the patient's systolic blood pressure but did exceed the patient's diastolic blood pressure (Table 1).

\section{Discussion}

Uterine tamponade balloons are emerging as a fertility sparing and lifesaving option in the management of PPH [6]. In 1992, Bakri 
introduced intrauterine balloon tamponade for the treatment of obstetric hemorrhage during cesarean delivery [7]. Recent literature has shown an overall success rate of $75 \%$ to $94 \%$ at avoiding embolization or hysterectomy. The principle of balloon tamponade therapy is to fill the uterine cavity to control bleeding with pressure [8]. This is achieved as a result of uniform pressure over the open sinuses of the uterus, the pressure on the blood vessel is greater than the pressure within the vessel. If pressure is applied for long enough, the blood will clot and form a permanent seal [9]. In non-uterine systems in which bleeding is successfully counteracted by balloon tamponade, relative ely low ILPs are used. For example, only $25-30 \mathrm{mmHg}$ is required when using the Sengstaken-Blakemore tube in the oesophagus [10].In the bladder, a pressure equal to the diastolic arterial pressure', approximately 75-80 $\mathrm{mmHg}$, is required [11]. However, there does not appear to be any published experimental recordings of postpartum uterine pressures or data on the actual pressures required to stop bleeding from a postpartum hemorrhage uterus.

Our study sought to detect the intraluminal pressure to establish positive tamponade test in vivo, assess the mechanism to explain the attainment of a positive tamponade test. We found that Bakri balloon could exert a positive tamponade test at $405 \pm 47$ $\mathrm{ml}$ filling volumes, which was recommended by the manufacturers and other scholars. As noted previously, the intraluminal pressure can be impacted by external forces, such as fundal contractility. We found a linear regression relationship between the intraluminal pressure and the balloon volume in vitro, the linear model is $y=31.033+0.768 x$, ( $y$ : intraluminal pressure , $x$ : liquid volume $)$. Our results were similar to Kathleen M [12] and Georgiou [2]. Georgiou had published a series of intraluminal pressure readings during the establishment of uterine tamponade using the Bakri balloon, he found a curvilinear relationship between the intraluminal pressure and the balloon volume in vitro and higher pressures in vivo, but with the limitations based on only two cases in vivo [2]. We obtained 30 cases, including 12 cases combined with other surgical hemostasis techniques, these cases could provide reproducibility of the results, were able to demonstrate uterine activity simultaneously independently from ILP recordings.

We found that when positive tamponade test established, ILPs increased in vivo and was higher than in vitro, the average difference is $39 \pm 12 \mathrm{mmHg}$. which was almost equal to uerine spiral artery vasculature pressure [13]. We also found the actual ILPs did not exceed the patient's systolic blood pressure but did exceed the patient's diastolic blood pressure to achieve a positive tamponade test, which was in accordance with C Georgiou's conclusion [2]. The relationship between ILP, intraluminal volume, systolic/ diastolic blood pressures and bleeding cessation were inconsistent. We speculated that uterine wall compliance with the intrauterine balloon which inflated by some volume liquid, the good fitness of the balloon- uterine interface, stimulate uterine muscle fibers that form a figure of eight, result in uterine contraction and placenta bed vasculature hemostasis after delivery. Thus, the clinical endpoint of a positive tamponade test may be a function of the pressure exerted by uterine contraction combined with the ball boon liquid luminal pressure, which could explain the difference between the ILPs in vivo and in vitro.

So, we proposed that to establish a positive tampona de test, some prerequisites should exist. The first prerequisite is that the uterine is enlarged and atonic, which could not able to counteract an increase in uterine cavity volume and could not expel the inserted balloon. We once had a postpartum hemorrhage patient, with part of placenta retention, vaginal bleeding with the uterine in good contraction, Bakri balloon was tried three times but expelled from the uterus immediately, at last, uterine artery embolization worked. The second prerequisite is that a good fitness of balloon-uterine interface, the balloon should be in some kind of material, with some tension, elastic, so that the effect of contact by the balloon at the uterine surface could elicit uterine secondary contration. The third prerequisite is the ILPs should be higher in vivo than in vitro at the corresponding liquid volume, the increased value is worked out by the uterus secondary contraction force combined with the inside balloon and is almost equal to the uterine spiral artery vasulature pressure ,30-40mmHg. During pregnancy, the spiral artery in the endometrium and myometrial segments is the main blood supply to the uteroplacenta, the vasculature pressure is $20-40 \mathrm{mmHg}$ [13]. We proposed the value of increased ILPs could be used to help to determine whether the positive tamponade test set up.

\section{Conclusion}

After a positive tamponade test was established, the intralum inal pressure within the tamponade balloon increased in vivo than in vitro, the difference value was roughly equal to the uterine spiral artery vasculat ure pressure.

\section{Limitations}

The observed ILPs readings had a number of limitations. The major limitation was that because all the patients were in rescue condition, we did not have enough time to measure the ILPs in the process of inserting the balloon, we did detect the ILPs after the establishment of positive tamponade test. The other limitation is that the liquid volume of positive tamponade test was determined by experience.

\section{Acknowledgment}

we acknowledge every author's hard and tedious work.

\section{Human Rights}

The work described has been carried out in accordance with The Code of Ethics of the World Medical Association (Declaration of Helsinki) for experiments involving humans; Uniform Requirements for manuscripts submitted to Biomedical journals. We stated that informed consent was obtained for experimentation with human subjects. The privacy rights of human subjects were always be observed. 


\section{References}

1. Georgiou C (2009) Balloon tamponade in the management of postpartum haemorrhage: a review. BJOG 116(6): 748-757.

2. Georgiou C (2010) Intraluminal pressure readings during the establishment of a positive 'tamponade test' in the management of postpartum haemorrhage. BJOG 117(3): 295-303.

3. Condous GS, Arulkumarah S, Symonds I (2003) The 'tamponade test' in the management of massive postpartum hemorrhage. Obstet Gynecol 101(4): 767-72.

4. Bakri YN, Amri A, Abdul Jabbar F (2001) Tamponade-balloon for obstetrical bleeding. Int J Obstet Gynecol 74(2): 139-142.

5. Cho Y, Rizvi C, Uppal T, Condous G (2008) Ultrasonographic visualization of balloon pla cement for uterine tamponade in massive primary postpartum haemorrhage. Ultrasou nd Obstet Gynecol 32(5): 711-713.

6. Chapter 89 Balloon tamponade and uterine packing for major PPH.

7. Bakri YN (1992) Uterine tamponade-drain for hemorrhage secondary to placenta previa-accreta. Int J Gynaecol Obstet 37(4): 302-303.

\section{ISSN: 2574-1241}

DOI: 10.26717/BJSTR.2019.15.002735

Liang Bai. Biomed J Sci \& Tech Res

(C) This work is licensed under Creative

Submission Link: https://biomedres.us/submit-manuscript.php
8. Dabelea V, Schultze PM, Mc Duffie RS (2007) Intrauterine balloon tamponade in the mana ge ement of postpartum hemorrhage. Am J Perinatol 24(6): 359-364

9. Akhter S, Begum MR, Kabir J (2005) Condom hydrostatic tamponade for massive postpartum hemorrhage. Int J Gynaecol Obstet 90(2): 134-135.

10. Sengstaken RW, Blakemore AH (1950) Balloon tamponade for the control of hemorrhage from esophageal varices: Sengstaken and Blakemore. Ann Surg 131(5): 781-789.

11. Gittes RF (1979) Tumors of the bladder. In: Harrison JH, (Eds.), Campbell's Urology, ( $4^{\text {th }}$ edn.), WB Saunders Chapter 29, Boston MA, USA 2: 1064 .

12. Kathleen M Antony, Diana A Racusin, Michael A Belfort, Gary A (2017) Dildy III. Under Pressure: Intraluminal Filling Pressures of Postpartum Hemorrhage Tamponade Balloons. American Journal of Perinatology Reports 7(2): 586-592

13. F Gary Cunningham, Kenneth J Leveno, Steven L Bloom, Jodi S Dashe, Barbara L Hoffman, et al. (2012) Williams Obstetric 25e: 90-94.

$\begin{array}{ll}\text { BIOMEDICAL } & \text { Assets of Publishing with us } \\ \text { RESEARCHES } & \text { - Global archiving of articles } \\ & \text { - Immediate, unrestricted online access } \\ & \text { - Rigorous Peer Review Process } \\ & \text { - Authors Retain Copyrights }\end{array}$

